\title{
Efficacy of Intravenous Ferric Carboxymaltose in Patients with Acute Post-Operative Anemia after Colorectal Cancer Surgery
}

\author{
Young Ju Oh, M.D., Dae Hee Pyo, M.D., Jung Kyong Shin, M.D., Yoon Ah Park, M.D., Jung Wook Huh, M.D., \\ Hee Cheol Kim, M.D., Seong Hyeon Yun, M.D., Woo Yong Lee, M.D., Yong Beom Cho, M.D., Ph.D. \\ Department of General Surgery, Samsung Medical Center, Sungkyunkwan University, Seoul, Korea
}

\begin{abstract}
Purpose: This study evaluated the efficacy of administering intravenous ferric carboxymaltose (Ferinject ${ }^{\circledR}$ ) in patients with acute post-operative anemia after colorectal surgery.

Materials and Methods: Patients with colorectal cancer, who underwent colectomy from January 2017 to July 2019 at the Samsung Medical Center, were retrospectively reviewed. Depending on their body weight, patients were administered $500 \mathrm{mg}$ or $1000 \mathrm{mg}$ of ferric carboxymaltose (ferric carboxymaltose group, 92 patients). The primary outcome evaluated was serum hemoglobin level on post-operative day 21 . The secondary outcome was the number of hemoglobin responders, defined as the proportion of patients with normalized serum hemoglobin on post-operative day 21 (hemoglobin level of $12 \mathrm{~g} / \mathrm{dL}$ or more for male, $11 \mathrm{~g} / \mathrm{dL}$ or more for female), and a serum hemoglobin increase of $2 \mathrm{~g} / \mathrm{dL}$ or more from baseline (day 0 ).

Results: Of the 560 patients who underwent colectomy, 92 patients (median age, 67 years; women 59.8\%; mean baseline hemoglobin level $10.7 \mathrm{~g} / \mathrm{dL}$ ) received intravenous ferric carboxymaltose. Compared with the no-ferric carboxymaltose group, patients in the ferric carboxymaltose group experienced significantly greater improvement in serum hemoglobin level (post-operative day 5: $8.9 \mathrm{~g} / \mathrm{dL}, \mathrm{P}=0.09$; post-operative day $21: 11.7 \mathrm{~g} / \mathrm{dL}, \mathrm{P}=0.006$ ). The increase in hemoglobin levels was significantly greater in the ferric carboxymaltose group $(2.7 \mathrm{~g} / \mathrm{dL})$ than the no-ferric carboxymaltose group $(2.1 \mathrm{~g} / \mathrm{dL})(\mathrm{P}=0.002)$.

Conclusion: Findings of this study indicate a better outcome after administering intravenous ferric carboxymaltose, which results in continuous increase in the levels of hemoglobin during the early post-operative period after colorectal cancer surgery.
\end{abstract}

Keywords: Hemoglobin, Anemia, Colon cancer, Post-operative, Ferric carboxymaltose

\section{INTRODUCTION}

Colorectal cancer is the third most common cancer in the world, following lung and breast cancers, and it has the second-highest death rate next to lung cancer.[1] Regardless of gender or age, colorectal cancer incidence is increasing worldwide, and the treatment and management of pre and postoperative symptoms and improving quality of life are becoming essential.[2] An examination of patients diagnosed with anemia-related and gastrointestinal bleeding revealed that up to $75 \%$ of cases were related to underlying cancers, with up to $69 \%$ of anemias caused by colorectal cancers.[35] Anemias are caused by increased hepcidin production from systemic tumor inflammation, iron deficiencies resulting

Received April 23, 2020; Revised July 19, 2020; Accepted August 11, 2020

Corresponding author: Yong Beom Cho

E-mail gscyb@skku.edu (iD) ORCID https://orcid.org/0000-0002-9944-4706

(i) \& This is an Open Access article distributed under the terms of the Creative Commons Attribution Non-Commercial License (http://creativecommons.org/licenses/ by-nc/4.0), which permits unrestricted non-commercial use, distribution, and reproduction in any medium, provided the original work is properly cited. 
from gastrointestinal bleeding, or nutritional deficiencies, or frequent chemotherapy.[2-4,6-8] Additionally, anemia can be an independent predictor for oncological outcome and survival rate.[9]

Anemia treatment commonly involves taking iron supplements or blood transfusion. Oral iron supplements, when consumed over a long time, can directly irritate the stomach lining, and have a low compliance rate caused by side effects such as constipation.[8] According to several retrospective studies, patients who received transfusions after colorectal cancer surgery had higher anemia recurrence rates and infection complications, which indicate interest in intravenous iron administration.[2,10-12] Some comparative clinical trials demonstrate how intravenous iron showed faster and longer-lasting curative effects compared to oral iron supplements, $[12,13]$ and an animal study showed oral iron supplements increased intestinal inflammation and incidence rate of colorectal cancers.[14] Therefore, this study analyzed the

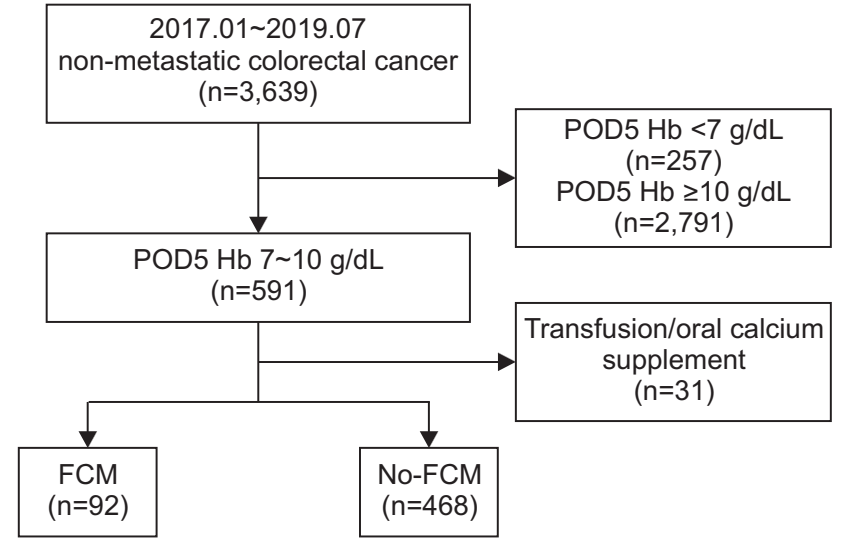

Fig. 1. Flow of patients through the study of ferric carboxymaltose for postcolectomy anemia.

Table 1. Baseline characteristics for patients with acute postoperative anemia following colectomy receiving ferric carboxymaltose (FCM) vs. no-ferric carboxymaltose (no-FCM)

\begin{tabular}{|c|c|c|c|}
\hline & FCM $(n=92)$ & no-FCM $(n=468)$ & $\mathbf{P}$ \\
\hline Sex, n (\%) & & & 0.657 \\
\hline Female & $55(59.8)$ & $265(56.6)$ & \\
\hline Male & $30(40.2)$ & $203(43.4)$ & \\
\hline Age, median (IQD) & $67(56 \sim 77)$ & $63(54 \sim 74)$ & 0.045 \\
\hline BMI, mean (SD), $\mathrm{kg} / \mathrm{m}^{2}$ & $23.5(3.6)$ & $23.3(3.6)$ & 0.576 \\
\hline Preoperative Hct, mean (SD), \% & $34.0(4.1)$ & $34.7(4.4)$ & 0.174 \\
\hline Preoperative $\mathrm{Hb}$, mean (SD), g/dL & $10.7(1.6)$ & $11.0(1.7)$ & 0.159 \\
\hline Tumor location, $\mathrm{n}(\%)$ & & & 0.075 \\
\hline Colon & $63(68.5)$ & $364(77.8)$ & \\
\hline Rectum & $29(31.5)$ & $104(22.2)$ & \\
\hline Type of operation, n (\%) & & & 0.463 \\
\hline MIS (Lap, SILS, Robot) & $74(80.4)$ & $394(84.2)$ & \\
\hline Open & $18(19.6)$ & $74(15.8)$ & \\
\hline Name of operation, $\mathrm{n}(\%)$ & & & 0.120 \\
\hline Colectomy & $51(33.4)$ & $231(49.4)$ & \\
\hline AR/LAR & $38(41.3)$ & $232(49.6)$ & \\
\hline APR & $3(3.3)$ & $5(1.1)$ & \\
\hline EBL, n (\%) & & & 0.101 \\
\hline$<100 \mathrm{~mL}$ & $47(51.1)$ & $263(56.2)$ & \\
\hline $100 \mathrm{~mL} 5500 \mathrm{~mL}$ & $45(48.9)$ & $190(40.6)$ & \\
\hline$\geq 500 \mathrm{~mL}$ & $0(0)$ & $15(3.2)$ & \\
\hline AJCC 7th staging, $\mathrm{n}(\%)$ & & & 0.001 \\
\hline I & $34(37.0)$ & $92(19.7)$ & \\
\hline II & $29(31.5)$ & $193(41.2)$ & \\
\hline III & $29(31.5)$ & $183(39.1)$ & \\
\hline
\end{tabular}

Hct = hematocrit; $\mathrm{Hb}$ = hemoglobin; MIS = minimally invasive surgery; Lap = laparoscopic surgery; SILS = single incision laparoscopic; $\mathrm{AR}=$ anterior resection; $\mathrm{LAR}=$ low anterior resection; $\mathrm{APR}=$ abdominoperineal resection; $\mathrm{EBL}=\mathrm{estimate}$ blood loss. 
effects of intravenous iron (Ferric Carboxymaltose, Ferinject ${ }^{\circledR}$ ) on acute anemias after colorectal cancer surgery.

\section{MATERIALS AND METHODS}

From January 2017 to July 2019, an individual retrospective chart analysis was performed on 3,717 patients who underwent colectomy, anterior resection, lower anterior resection, or abdominoperineal resections for nonmetastatic primary colorectal cancers. Acute anemia was defined by hemoglobin levels between $7 \mathrm{~g} / \mathrm{dL}$ and $10 \mathrm{~g} / \mathrm{dL} 5$ days after surgery. In this study, patients were excluded if their hemoglobin levels were below $7 \mathrm{~g} / \mathrm{dL}$ ( 257 patients), did not meet the definition of anemia with hemoglobin levels above 10 $\mathrm{g} / \mathrm{dL}$ (2,791 patients), or took oral iron supplements upon discharge (31 patients). Out of the initial number of patients, 560 were recruited for the study (Fig. 1). Approval by the appropriate Institutional Review Boards was obtained.

Intravenous administration of iron was determined by the operating physicians' preferences and decisions. If serum hemoglobin levels 5 days after surgery were between $9 \mathrm{~g} / \mathrm{dL}$ $10 \mathrm{~g} / \mathrm{dL}, 500 \mathrm{mg}$ of intravenous iron was administered. If serum hemoglobin level was below $9 \mathrm{~g} / \mathrm{dL}$, then $1000 \mathrm{mg}$ of intravenous iron was administered. On the first outpatient visit (21 days after surgery), the follow-up serum hemoglobin level was checked and set as the primary outcome. A normalized serum hemoglobin level determined if this was above $12 \mathrm{~g} /$ $\mathrm{dL}$ for men and $11 \mathrm{~g} / \mathrm{dL}$ for women. The responder which was set as the secondary outcome included those with normalized levels or if serum hemoglobin increased by more than $2 \mathrm{~g} / \mathrm{dL}$ after intravenous iron administration.

A chi-squared test compared the proportion of the grouped variables with and without intravenous iron administration, and the student's t-test compared the average of the continuous variables. Meanwhile, a box graph analysis was

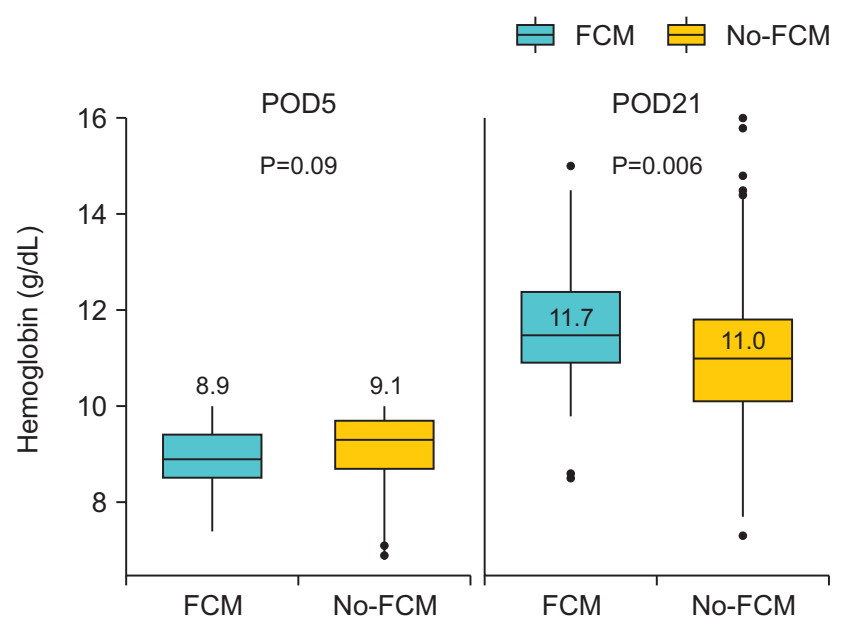

Fig. 2. Hemoglobin levels among patients receiving ferric carboxymaltose vs. no-ferric carboxymaltose at POD 5 and POD 21. performed to visually compare the two groups using R 3.53 and $R$ studio 1.2.1. Statistical significance was set at $P=0.05$.

\section{RESULTS}

In this study, 560 patients were divided into two groups, determined by who received intravenous iron. In the group that received intravenous iron, 55 were female $(59.8 \%)$, and 37 were male $(40.2 \%)$. Meanwhile, 265 female patients $(56.6 \%)$ and 203 male patients $(43.3 \%)$ did not receive intravenous iron. The median age was significantly higher in the patients receiving intravenous iron (67 vs. 63 years old, $\mathrm{P}=0.045)$, and there was no significant difference between the two groups' pre-operative average hemoglobin and hematocrit (Table 1).

Based on the 7th edition of the American Joint Committee on Cancer's (AJCC's) colorectal cancer classifications, the percentage of patients with stage 1 cancer was higher in those receiving intravenous iron compared to those without intravenous iron administration $(37.0 \%$ vs. $19.7 \%, \mathrm{P}=0.001)$. The percentage of patients with stage 2 or 3 cancers were lower in those receiving intravenous iron compared to those without intravenous iron administration $(31.5 \%$ vs. $41.2 \%$; 31.5 vs. $39.1 \%$, respectively, $\mathrm{P}=0.001$ ) (Table 1 ).

There was no significant difference between the two groups' hemoglobin levels 5 days after surgery, which were $8.9 \mathrm{~g} / \mathrm{dL}$ for the group with intravenous iron administration and $9.1 \mathrm{~g} / \mathrm{dL}$ for the group without intravenous iron administration. However, hemoglobin levels 21 days after surgery were significantly higher in the group with intravenous iron administration $(11.7 \mathrm{~g} / \mathrm{dL})$ than in the group without intravenous iron administration (11.0 g/dL; $\mathrm{P}=0.006)$ (Fig. 2). The amount of hemoglobin increase was also significantly higher for the group with intravenous iron $(2.7 \mathrm{~g} / \mathrm{dL})$ than in the group without intravenous administration $(2.1 \mathrm{~g} / \mathrm{dL}$,

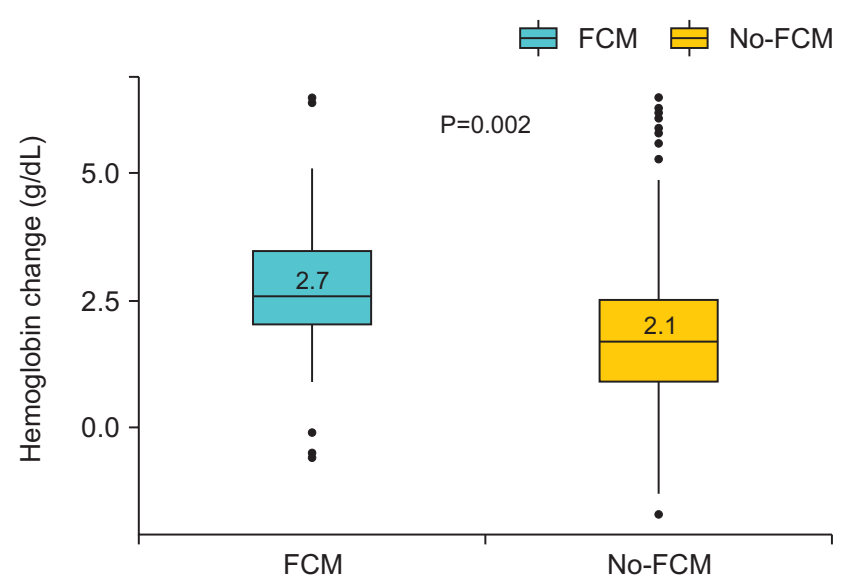

Fig. 3. Hemoglobin Changes Among Patients Receiving Ferric Carboxymaltose vs. no-Ferric Carboxymaltose at POD 5 and POD 21. 
Table 2. Comparison of outcomes between receiving ferric carboxymaltose (FCM) vs. no-ferric carboxymaltose (no-FCM)

\begin{tabular}{|c|c|c|c|}
\hline & FCM $(n=92)$ & no-FCM $(n=468)$ & $\mathbf{P}$ \\
\hline$\triangle \mathrm{Hb}$ (POD21-POD5), mean (SD), g/dL & $2.7(1.3)$ & $2.1(1.3)$ & 0.002 \\
\hline Responder, n (\%) & & & $<0.0001$ \\
\hline$(+)$ & $77(83.7)$ & $257(54.9)$ & \\
\hline$(-)$ & $15(16.3)$ & $211(45.1)$ & \\
\hline $\mathrm{Hb}$ increase from baseline, $\mathrm{n}(\%)$ & & & $<0.0001$ \\
\hline$\geq 2 \mathrm{~g} / \mathrm{dL}$ & $71(77.2)$ & $193(41.2)$ & \\
\hline$<2 \mathrm{~g} / \mathrm{dL}$ & $21(22.8)$ & $275(58.8)$ & \\
\hline Normalized at POD 21, n (\%) & $68(73.9)$ & $237(50.6)$ & $<0.0001$ \\
\hline
\end{tabular}

$\mathrm{Hb}=$ hemoglobin; $\mathrm{POD}=$ Post-op day.

$\mathrm{P}=0.002)$ (Fig. 3).

The variables that affected the change in hemoglobin levels 5 and 21 days after surgery in the two groups were examined and tabulated in Table 2. The percentage of the patients in the responder group, which included those with more than 2 $\mathrm{g} / \mathrm{dL}$ increase in absolute hemoglobin levels and patients with normalized hemoglobin levels 21 days after surgery were higher than in patients with intravenous iron administration.

\section{DISCUSSION}

Due to the rise of early colorectal cancer diagnosis using colonoscopy in recent years, the number of surgeries for early-stage colorectal cancer is increasing, which, in turn, increases long-term survival rates. Consequently, complications that affect the quality of life, such as anemia, nutrition, and metabolism, are becoming more important health issues. One treatment option for anemia involves consuming oral iron supplements chronically, but several studies showed that iron absorption was slow and caused numerous gastrointestinal side effects. Another treatment option involves blood transfusion, but some patients refused due to religious reasons, and others showed hypersensitivity, which necessitated pretreatment.[3] Previous studies showed that intravenous iron administration one week before surgeries drastically decreased the need for postoperative transfusion, $[15,16]$ and another study showed intravenous iron infusion was associated with a decreased necessity for transfusion and shorter stays in the hospital.[3] Therefore, this study evaluated the changes in hemoglobin levels in patients with low hemoglobin levels after colorectal cancer surgery based on intravenous iron administration. The results show that intravenous iron administration significantly increased hemoglobin levels 21 days after surgery and was associated with a higher percentage of patients with normalized hemoglobin levels. Through these findings, intravenous iron administration decreased anemia development in patients who underwent colorectal cancer surgery and reduced the need for transfusions. This retrospective comparison study, performed in one institution involving a much larger number of patients than seen in previous studies, demonstrated the positive therapeutic effect of intravenous iron administration in patients with anemia after colorectal cancer surgery.

Cancers in the digestive system, such as colorectal and gastric cancers, have a high risk of developing anemias due to various mechanisms, including gastrointestinal bleeding and consumption due to inflammatory response, which increases further after surgeries. The Farric Carboxymaltose for Acute Isovolemic Anemia Following Gastrectomy (FAIRY) clinical trial was a 3-year prospective randomized study. It demonstrated intravenous iron administration was effective in patients with postoperative anemia 12 weeks after gastrectomy; the number of patients with normalized or improved hemoglobin levels was higher in those who received intravenous iron administration than those who did not (92.2 vs. 54.0\%, $\mathrm{P}=0.001) .[4]$ In addition to the hemoglobin level, patients with intravenous iron administration had significantly higher levels of ferritin and total iron-binding capacity (TIBC) and a significantly lower percentage use alternative anemia treatment options, including transfusion or oral iron supplements.

In this study, we applied the FAIRY trial's definition of hemoglobin responders to the patients after colorectal cancer surgeries to find the effect of intravenous iron administration, which was based on the hemoglobin levels at week 12. Meanwhile, the current study considered the effects of chemotherapy on the development of anemia. The patients' hemoglobin levels at week 3, or before the start of chemotherapy, were used to define the responder group. Therefore, further studies are needed to determine whether intravenous iron administration can be continued two or three months after colorectal cancer surgeries.

There are several limitations in this study. First, it is a retrospective study, with possible bias in patient selection. Second, aside from iron deficiency anemia (IDA), anemias can develop from other causes such as anemias of chronic diseases. However, this study categorized and assessed patients based only on their hemoglobin levels. Without TIBC or ferric acid levels to diagnose IDA, anemia treatment evaluation is not straightforward. Third, the study did not evaluate the effects of hemoglobin level improvement on the quality of life and 
the oncologic prognosis after three weeks. Last, Ferinject ${ }^{\circledR}$, the intravenous iron used in the study, was not covered by patients' health insurance and was costlier than oral iron supplements or transfusion treatments. No cost-benefit analysis was conducted to determine whether the therapeutic effect of Ferinject ${ }^{\circledR}$ outweighs its cost.

This study reported on the positive effects of intravenous iron on anemia after colorectal cancer surgery. Therefore, future studies can expect better-designed, prospective randomized controlled trials based on the results.

\section{CONCLUSION}

This study determined that intravenous iron administration was an effective and safe treatment method for patients with anemias after colorectal cancer surgeries. Additional studies, including prospective randomized controlled studies, can evaluate the long-term effects of intravenous iron administration and determine if it has a positive effect on the quality of life or oncologic prognosis.

\section{CONFLICTS OF INTEREST}

The authors of this manuscript have no conflicts of interest to disclose.

\section{ORCID}

Yong Beom Cho, https://orcid.org/0000-0002-9944-4706

\section{REFERENCES}

1. Bray F, Ferlay J, Soerjomataram I, Siegel RL, Torre LA, Jemal A. Global cancer statistics 2018: GLOBOCAN estimates of incidence and mortality worldwide for 36 cancers in 185 countries. CA Cancer J Clin 2018;68:394-424.

2. Wu HL, Tai YH, Lin SP, Chan MY, Chen HH, Chang KY. The impact of blood transfusion on recurrence and mortality following colorectal cancer resection: a propensity score analysis of 4,030 patients. Sci Rep 2018;8:13345.

3. Calleja JL, Delgado S, del Val A, Hervás A, Larraona JL, Terán Á, et al.; Colon Cancer Study Group. Ferric carboxymaltose reduces transfusions and hospital stay in patients with colon cancer and anemia. Int J Colorectal Dis 2016;31:543-51.

4. Kim YW, Bae JM, Park YK, Yang HK, Yu W, Yook JH, et al.; FAIRY Study Group. Effect of intravenous ferric carboxymaltose on hemoglobin response among patients with acute isovolemic anemia following gastrectomy: the FAIRY randomized clinical trial. JAMA 2017;317:2097-104.
5. Aapro M, Österborg A, Gascón P, Ludwig H, Beguin Y. Prevalence and management of cancer-related anaemia, iron deficiency and the specific role of i.v. iron. Ann Oncol 2012;23:1954-62.

6. Ansarinejad N, Abbasi B, Sadat Rasul MS, Fardad F, Ramim T. The effectiveness of ferric carboxymaltose on the improvement of chronic iron deficiency anemia in patients with colon cancer: a controlled randomized clinical trial. Obstet Gynecol Cancer Res 2016;1:e8006.

7. Borstlap WAA, Buskens CJ, Tytgat KMAJ, Tuynman JB, Consten ECJ, Tolboom RC, et al. Multicentre randomized controlled trial comparing ferric(III)carboxymaltose infusion with oral iron supplementation in the treatment of preoperative anaemia in colorectal cancer patients. BMC Surg 2015;15:78.

8. Muñoz M, Gómez-Ramírez S, Martín-Montañez E, Auerbach $M$. Perioperative anemia management in colorectal cancer patients: a pragmatic approach. World J Gastroenterol 2014;20:1972-85.

9. Caro JJ, Salas M, Ward A, Goss G. Anemia as an independent prognostic factor for survival in patients with cancer: a systemic, quantitative review. Cancer 2001;91:2214-21.

10. Tartter PI, Quintero S, Barron DM. Perioperative blood transfusion associated with infectious complications after colorectal cancer operations. Am J Surg 1986;152:479-82.

11. Tartter PI. Blood transfusion and infectious complications following colorectal cancer surgery. Br J Surg 1988;75:78992.

12. Laso-Morales MJ, Vives R, Vallejo-Tarrat A, Caló N, ValleBeltran A, Pontes C. Single dose of intravenous ferric carboxymaltose infusion versus multiple fractionated doses of intravenous iron sucrose in the treatment of postoperative anaemia in colorectal cancer patients: study protocol for a randomised controlled trial. Trials 2019;20:23.

13. Schreiber S, Howaldt S, Schnoor M, Nikolaus S, Bauditz J, Gasché $C$, et al. Recombinant erythropoietin for the treatment of anemia in inflammatory bowel disease. N Engl J Med 1996;334:619-23.

14. Kulnigg S, Gasche C. Systematic review: managing anaemia in Crohn's disease. Aliment Pharmacol Ther 2006; 24:1507-23.

15. Muñoz M, Gómez-Ramírez S, Cuenca J, García-Erce JA, Iglesias-Aparicio D, Haman-Alcober S, et al. Very-shortterm perioperative intravenous iron administration and postoperative outcome in major orthopedic surgery: a pooled analysis of observational data from 2547 patients. Transfusion 2014;54:289-99.

16. Bisbe E, García-Erce JA, Díez-Lobo Al, Muñoz M; Anaemia Working Group España. A multicentre comparative study on the efficacy of intravenous ferric carboxymaltose and iron sucrose for correcting preoperative anaemia in patients undergoing major elective surgery. $\mathrm{Br} \mathrm{J}$ Anaesth 2011;107:477-8. 